\title{
Abdominal migraine in childhood: a review
}

REVIEW

This article was published in the following Dove Press journal:

Pediatric Health, Medicine and Therapeutics

7 August 2014

Number of times this article has been viewed

Beatrice Scicchitano'

Gareth Humphreys'

Sally G Mitton²

Thiagarajan Jaiganesh'

'Children's Emergency Department, 2Department of Paediatric Gastroenterology, St Georges Hospital, St Georges Healthcare NHS Trust, Tooting, London, United Kingdom
Correspondence: Thiagarajan Jaiganesh Children's Emergency Department, St Georges Hospital, St Georges Healthcare NHS Trust, Blackshaw Road, Tooting, London SWI7 0QT, United Kingdom

Email thiagarajan.jaiganesh@stgeorges. nhs.uk
Abstract: The childhood condition of abdominal migraine has been described under many different synonyms, including "abdominal epilepsy", "recurrent abdominal pain", "cyclical vomiting syndrome", and "functional gastrointestinal disorder". In the early literature, abdominal migraine is included in the "childhood periodic syndrome", first described by Wyllie and Schlesinger in 1933. Abdominal migraine has emerged over the last century as a diagnostic entity in its own right thanks to the development of well defined diagnostic criteria and its recent inclusion in the International Headache Society's Classification of Headache disorders. Despite this progress, little is known about the pathophysiology of the condition, and the treatment options are poorly defined. Here we summarize the recent literature, with particular focus on establishing the diagnosis of abdominal migraine and its pathophysiology, and suggest an approach to management.

Keywords: abdominal migraine, recurrent abdominal pain, abdominal epilepsy, cyclical vomiting

\section{Introduction}

Abdominal migraine in children is a condition characterized by recurrent episodes of periumbilical pain severe enough to interfere with daily activities for which no organic cause is found. It is associated with vasomotor and gastrointestinal symptoms, including anorexia, nausea, vomiting, and pallor. It is also distinguished by an absence of symptoms in the intervening periods. Attacks typically last between one and 72 hours and are recurrent within a 12 -month period. ${ }^{1}$ While the prevalence of recurrent abdominal pain in children ranges from $9 \%$ to $15 \%,{ }^{2}$ the estimated prevalence of abdominal migraine as a subset of this is within the range of $2.4 \%$ and $4.1 \%{ }^{1,3}$ The mean age of onset is 7 years, although it has been described in infants and adults, with females affected more frequently than males. ${ }^{4}$

Abdominal migraine is usually diagnosed at the age of 3-10 years, ${ }^{3}$ and typically follows a benign course into adulthood. Early studies into the childhood periodic syndromes by Cullen and MacDonald 5 found that childhood abdominal migraine typically evolved into migraine headaches in adulthood. There have also been several studies linking abdominal migraine to migraine headache in later life and a family history of migraine. ${ }^{1,6}$ These findings have been endorsed by the International Headache Society's definition of abdominal migraine, ${ }^{7}$ which includes a reference to migraine headache and has implications for prognosis and management strategies.

Children with recurrent abdominal pain are at risk of impairment of their social and educational development, in addition to placing a significant burden on community's 
health care resources. ${ }^{2}$ Despite the existence of diagnostic criteria, it is likely that abdominal migraine remains underdiagnosed in the community. Our objective is to summarize the investigations and decision-making tools available to make the diagnosis of abdominal migraine in children. Furthermore, we aim to summarize the evidence supporting a link between abdominal migraine and other childhood disorders, and the current treatment strategies available for treatment of this condition.

\section{Pathophysiology}

Despite significant progress in defining abdominal migraine in the literature over the past century, there has been little development of our understanding of its pathophysiology. Given the strong relationship between abdominal migraine, migraine headache, and cyclical vomiting, it has been suggested that they share a common cause, but this has yet to be defined. ${ }^{8}$ In particular, the similarities between migraine headache and abdominal migraine regarding precipitants, associated symptoms, demographics, and treatment modalities suggest a shared etiology. ${ }^{1}$ Many of the treatment options proposed for abdominal migraine have been based on its well documented association with migraine headache rather than on the basis of its underlying pathophysiology. While the etiology of migraine headache has been widely accepted, the ability to expand these mechanisms to provide an explanation of abdominal migraine symptoms has been difficult to achieve.

While the pathophysiology of abdominal migraine remains largely unknown and untested, there are several hypotheses for the functional gastrointestinal disorders as a group, which include abdominal migraine, irritable bowel syndrome, and cyclical vomiting. These theories have included autonomic instability, disturbance in the hypothalamic-pituitary-adrenal axis, and altered motility of the gut wall. ${ }^{4}$ Recently, possible genetic influences and differences in immune and neuronal structures within the bowel mucosa have been put forward as potential underlying physiological mechanisms for the disorders. ${ }^{9,10}$ observed significant differences in gut mucosal permeability between patients with abdominal migraine and controls. They proposed that as mucosal permeability increased, symptoms worsened, and, conversely, as patients improved, their mucosal permeability decreased. The authors felt that although the observation may not explain the mechanism of pain in abdominal migraine, it may give rise to new avenues of treatment for the disorder.

The predominant theory in the literature with increasing support is that of visceral hypersensitivity to distension in response to abnormalities in neurophysiology at the level of the gut, spinal cord, or higher cortical systems. ${ }^{11}$ The pathophysiology behind this model is that there is extensive two-way communication between the enteric nervous system and the central nervous system, which strengthens the hypothesis that stress stimulates the central nervous system and gives rise to an abnormal effect on the gastrointestinal system through dysregulation of neuropeptide and neurotransmitter release. ${ }^{4,12}$ Collins and Thomas ${ }^{12}$ proposed a biopsychosocial approach to understanding the development of chronic abdominal pain. The authors surmised that the interplay between psychological factors, altered gut motility, and abnormal visceral perception caused an alteration in the "brain-gut" axis linking the enteric nervous system and the central nervous system. None of the aforementioned theories have been studied in relation to abdominal migraine as a specific disease entity, but certainly warrant further investigation into their validity.

\section{Diagnosis and investigations}

The term abdominal migraine was utilized by Brans ${ }^{13}$ to describe periodic attacks of epigastralgia characterized by symptom-free intervals between episodes and a history of migraine in either the patient or his relative. Since that early description of the disorder, there have been several advances in defining abdominal migraine in addition to the founding of diagnostic criteria. A detailed description of abdominal migraine was first proposed by Symon and Russell ${ }^{14}$ In this study, children with recurrent abdominal pain and a family history of migraine were identified and studied in terms of their symptomatology and response to traditional migraine treatment. Subsequent application of these diagnostic features to a population of schoolchildren with recurrent abdominal pain in Aberdeen showed a prevalence of $4.1 \%$, which was consistent with previously documented prevalence rates for abdominal migraine. ${ }^{1}$ Dignan et $\mathrm{al}^{15}$ developed a comprehensive guideline for diagnosis of abdominal migraine (see Figure 1), which included the following exclusion criteria: mild symptoms not interfering with daily activities, burning pain, non-midline pain, symptoms suggestive of food allergy or other organic disease, attacks lasting less than one hour, or persistence of symptoms between attacks. ${ }^{15}$

In 2004, the International Headache Society included diagnostic criteria for abdominal migraine in the International Classification of Headache Disorders Second Edition (ICHD-II, Figure 2). ${ }^{7}$ In addition to these criteria, the presence of gastrointestinal or urinary tract disease must be excluded on the basis of history, physical examination, or investigations. Similar criteria were added to the Rome III Pediatric Criteria for Functional Gastrointestinal Disorders 
- Pain is severe enough to interfere with normal daily activities

- Pain is described as dull or sore in nature

- Pain is periumbilical or poorly localized

- Pain is associated with any two of the following:

- Anorexia

- Nausea

$\circ$ Vomiting

- Pallor

- Each attack lasts for at least one hour

- There is complete resolution of symptoms between attacks

- Attacks occur at least twice a year

- The diagnosis is excluded if any of the following is present:

- Mild symptoms not interfering significantly with daily activities

- Burning pain

- Non-midline abdominal pain

- Symptoms suggestive of food intolerance, malabsorption, or other gastrointestinal disease

- Attacks of less than one hour duration

- Persistence of symptoms between attacks

Figure I Criteria for diagnosis of abdominal migraine.

Note: Adapted by permission from BMJ Publishing Group Limited. Archives of Disease in Childhood. The prognosis of childhood abdominal migraine. F Dignan, I Abu-Arafeh, G Russell. 84:415-4|8. (c) 2001.15

in 2006 (Figure 3). ${ }^{16}$ The two classifications differ in several aspects, namely, the number of episodes required to confirm the diagnosis and the mention of periods of intervening wellness in the Rome III criteria. The Rome III criteria also classify other pediatric functional gastrointestinal disorders, such as cyclical vomiting, functional dyspepsia, irritable bowel syndrome, and functional constipation. A study of Norwegian children referred with nonorganic abdominal pain showed significant overlap between diagnoses based on the Rome III criteria, with $34 \%$ of children meeting criteria for more than one condition. ${ }^{17}$

A common thread among the three sets of diagnostic criteria discussed here is the absence of organic disease. The prevalence rates of recurrent abdominal pain vary between $0.5 \%$ and $19 \%$

- A At least five attacks fulfilling criteria $B-D$

- B Attacks of abdominal pain lasting 1-72 hours (untreated or unsuccessfully treated)

- C Abdominal pain has all of the following characteristics:

- Midline location, periumbilical or poorly localized

- Dull or "just sore" quality

- Moderate or severe intensity

- D During abdominal pain at least two of the following:
- Anorexia
- Nausea
$\circ$ Vomiting
- Pallor

- E Not attributed to another disorder

Figure 2 International classification of headache disorders second edition diagnostic criteria for abdominal migraine.

Note: Olesen J, The International Classification of Headache Disorders, Second Edition, Cephalalgia (24) Suppl I, pp. 9-160, copyright (c) 2004 by Sage Publications, reproduced with permission of SAGE. ${ }^{7}$
- Paroxysmal episodes of intense, acute periumbilical pain that lasts for one hour or more

- Intervening periods of usual health lasting weeks to months

- Pain interferes with normal activities

- Pain is associated with two or more of the following:

- Anorexia

- Nausea

- Vomiting

- Headache

- Photophobia

- Pallor

- No evidence of an inflammatory, anatomic, metabolic or neoplastic process considered that explains the patient's symptoms

- Criteria fulfilled two or more times in the preceding 12 months.

Figure 3 Rome III criteria for abdominal migraine.

Note: Copyright (C) 2006, Elsevier Limited. Reproduced from Gastroenterology, 130, Rasquin A, Di Lorenzo C, Forbes D, et al, Childhood functional gastrointestinal disorders: child/adolescent, 1527-1537, 2006, with permission from Elsevier Limited. ${ }^{16}$

based on community epidemiological studies. ${ }^{9}$ In their field survey of 1000 school children, Apley and Naish ${ }^{18}$ suggested that organic pathology was identifiable in only $8 \%-10 \%$ of children with recurrent abdominal pain. Therefore, determining the extent to which recurrent abdominal pain is investigated becomes a crucial factor in the diagnostic process. Given the extensive list of differential diagnoses for recurrent abdominal pain, the potential for exhaustive and costly diagnostic evaluation of patients is significant. The differential diagnosis of abdominal migraine must include inflammatory conditions, structural abnormalities, metabolic disorders, food intolerances, urinary tract pathology, and other functional gastrointestinal disorders. ${ }^{11,19,20}$ In an effort to identify children with recurrent abdominal pain in need of further investigation, physicians have often utilized so-called "red flag" signs and symptoms which increase the likelihood of underlying organic disease (Figure 4). ${ }^{12,21}$ While Catto-Smith and Ranuh ${ }^{22}$

- Change in growth patterns

- Recurrent unexplained fevers

- Pain radiating to the back

- Bilious emesis

- Visible or occult blood in stool

- Chronic diarrhea (lasting $>2$ weeks and $>20 \mathrm{~mL} / \mathrm{kg}$ per day)

- Mouth ulcers

- Dysphagia

- Rashes with no identifiable cause

- Nocturnal symptoms

- Anemia

- Pale mucus membranes

- Delayed puberty

- Family history of inflammatory bowel disease

- Joint swelling, tenderness or heat

- Organomegaly

- Localized abdominal tenderness, away from umbilicus

- Dysuria

Figure 4 Red flags suggestive of further investigation required. 
suggest a wide range of initial diagnostic tests in children presenting with possible abdominal migraine, other studies have identified only basic urine, blood, and stool analyses as recommended tests to exclude organic causes of recurrent abdominal pain. ${ }^{20}$ In our experience, we recommend a combination of blood, urine, stool, and an abdominal ultrasound as the initial investigations in an emergency setting. (Figure 5) If these are normal, then children should be referred to a pediatric gastroenterologist who may consider other invasive investigations if the diagnosis is still unclear (Figure 6).

\section{Clinical associations}

On multiple occasions in the literature, the condition we now call abdominal migraine is included in a general discussion of the childhood periodic syndromes; a group of conditions characterized by their episodic nature with symptom-free intervals. Other conditions included under this heading include migraine headaches, cyclical vomiting, benign paroxysmal torticollis, and benign paroxysmal vertigo of childhood. The literature suggests that these conditions are migraine subtypes and precursors to migraine headache, although only abdominal migraine, benign paroxysmal vertigo of childhood, and cyclical vomiting are mentioned in the International Classification of Headache Disorders Second Edition. ${ }^{7}$

One of the earliest papers to investigate the association between abdominal migraine and migrainous headaches was published in $1956 .{ }^{6}$ The authors of this case series investigated a total of 112 patients between the ages of 18 months and 15 years with symptoms of headache, recurrent vomiting, and recurrent abdominal pain in varying combinations based on the predominance of symptoms. It was found that over half the

Blood studies

- Full blood count

- Erythrocyte sedimentation rate

- C-reactive protein

- Electrolytes

- Urea and creatinine

- Glucose

- Liver function tests

- Amylase and lipase

- Celiac antibodies

- Pregnancy test

Urine and stool studies

- Urinalysis with microscopy, culture, and sensitivity

- Stool occult blood and microscopy

- Stool test for Helicobacter pylori antigen

Radiological studies

- Ultrasound of the abdomen and pelvis (may help with malrotation and superior mesenteric artery syndrome)

Figure 5 Initial diagnostic studies that should be considered in children presenting with possible cyclical vomiting or abdominal migraine in an emergency setting.
Radiological studies (rarely used due to significant radiation)

- Contrast study of upper gastrointestinal tract and small bowel

- Magnetic resonance imaging of brain

Endoscopy

- Esophagogastroduodenoscopy

- Colonoscopy with ileoscopy to exclude Crohn's disease (very invasive and rarely required)

Figure 6 Other diagnostic studies that could be considered in children presenting with possible cyclical vomiting or abdominal migraine by pediatric gastroenterology.

children (64\%) had one or two parents who suffered migraines and that the abdominal symptoms became less pronounced with advancing age. The authors also observed that travel sickness was a common, though not formally analyzed, complaint both in the children and their family members. ${ }^{6}$ This association was supported by further studies in $1983^{23}$ and $^{3}$ showed that a significant proportion of children with abdominal migraine or migraine also suffered motion sickness. A subsequent 1995 study demonstrated a prevalence rate of migraine headache amongst children with abdominal migraine twice more than twice that of the general childhood population (24\% versus $10.6 \%) .{ }^{1}$ Similarly the prevalence rate of abdominal migraine in children with migraine was over twice that of abdominal migraine in the general childhood population ( $9 \%$ versus $4.1 \%$ ). This same study also showed comparable trigger and relieving factors in addition to associated sensory and vasomotor symptoms between the two conditions. The link in prevalence between abdominal migraine and migraine headache has thus been clearly established in population studies with implications for both prognosis and treatment options.

While the clinical association between abdominal migraine and migraine headache has been well established, the link between this condition and the other periodic syndromes of childhood has been less observed. A 2010 population study by Arruda et al looked at the prevalence of symptoms suggestive of the childhood periodic syndromes in addition to the prevalence and relative risk of these symptoms in migraine headache relative to controls. The study found that recurrent abdominal pain was the most prevalent complaint (32.9\%) of the interictal symptoms investigated. In children suffering episodic migraine, the prevalence of abdominal pain was $58.6 \%$, with a relative risk of 2.6 (95\% confidence interval 1.9-3.3). This was second only to limb pain. The authors felt the results confirmed previous studies linking childhood periodic syndromes to migraine and migraine subtypes. ${ }^{24}$ The clinical association between abdominal migraine and the other periodic syndromes of children is likely to have implications for prognosis and treatment although these have not yet been established. 
It is clear that the diagnosis of abdominal migraine requires a careful and detailed history and physical examination in combination with selective and judicial diagnostic studies and utilization of validated diagnostic criteria. ${ }^{8}$ proposed a diagnostic algorithm for the assessment of children presenting with recurrent abdominal pain. We have modified this algorithm which reflects our current practice (Figure 7). This approach may be useful in achieving a timely diagnosis without the need for overly invasive and costly investigations.

\section{Management}

Little has been concluded about the treatment of abdominal migraine. Much like other "chronic" disease, perhaps the most important question to ask is, should abdominal migraine be treated ${ }^{25}$ Children are well between episodes of abdominal migraine but by definition pain lasts more than an hour when present and interferes with normal daily activities. The severity, frequency, and morbidity associated with episodes of abdominal migraine must be taken into account. This necessitates careful analysis of the potential benefits of different treatment options to be weighed against the risk of unpleasant side effects or possible harm. Given the episodic nature of abdominal migraine, the likely psychosocial factors involved and the lack of knowledge regarding its pathophysiology treatment options are varied, with both nonpharmacological and pharmacological alternatives.

\section{Nonpharmacological therapy}

Nonpharmacological therapy is frequently first-line in the treatment of abdominal migraine. Often a detailed explanation of the disease and its prognosis is all that is required in cases of mild abdominal migraine. In particular, families should be reassured that serious abdominal pathology is absent and that symptoms will likely subside with advancing age. ${ }^{25}$ Once children have been reassured and the migrainous nature of the pain has been explained, some children may be able to recall triggers for the pain. There is a minimal evidence

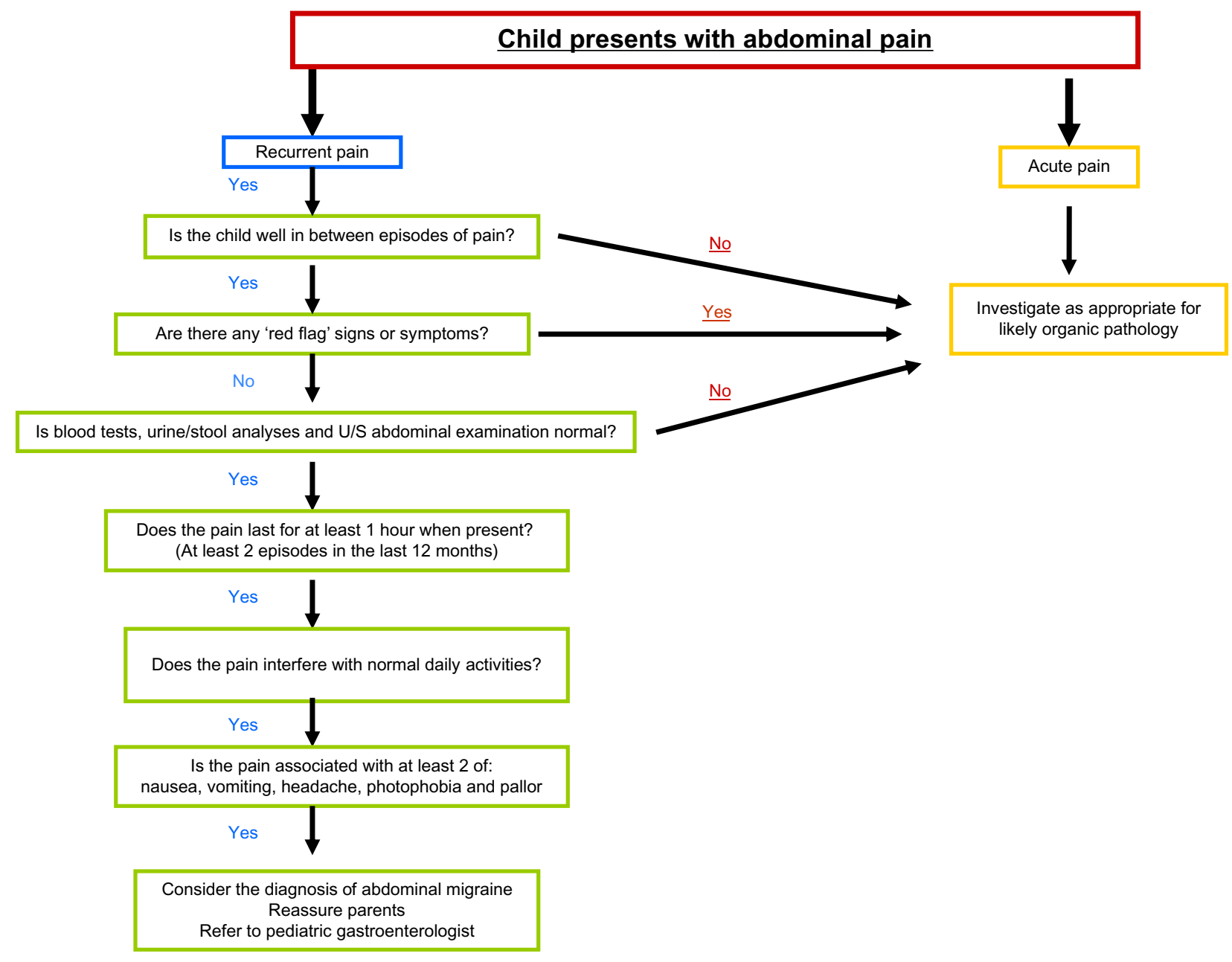

Figure 7 Modified diagnostic algorithm for the assessment of children presenting with recurrent abdominal pain. 
base for this, but from their experience, Russell et al ${ }^{25}$ suggested that triggers may include stress, travel, prolonged fasting, exposure to flickering lights, exercise, and alterations of sleep patterns. Avoidance of known or suspected triggers may improve the frequency or severity of attacks, although the evidence to support this theory is lacking.

Dietary modifications fall into three main groups, ie, increased fiber, reduced lactose, and a "few foods" diet, each with a paucity of evidence to support them. Feldman et $\mathrm{al}^{26}$ studied the effect of increasing fiber in the diet of children who had abdominal migraine. They reported a significant benefit of increased fiber in their study of 52 children. The authors concluded that $50 \%$ of children in the fiber group had a $50 \%$ reduction in the number of episodes of abdominal pain, compared with only $27 \%$ in the control group. Historically, a reduced lactose diet has been advocated for the treatment of abdominal migraine. Barr and Liebman ${ }^{27,28}$ demonstrated that a significant proportion of children with recurrent abdominal migraine had an abnormal lactose tolerance test and showed complete resolution of symptoms with a lactose-free diet. However, these children would not meet the definition criteria for abdominal migraine which excludes organic disease, and so eliminating lactose from the patient's diet is unlikely to produce a significant change in their symptoms.

Russell et al described the "few foods or "oligo-antigenic" diet as a dietary modification the authors applied within their own practice for children with abdominal migraine. ${ }^{25}$ The authors listed a number of foods, including chocolate, citrus fruits, caffeine, cheese, and colorings that may trigger abdominal migraine and should thus be avoided. The "few foods" diet treatment requires careful supervision by a dietician and is reserved for those children who have frequent attacks ( $>2$ per week). The child is commenced on a diet consisting of a very small number of foods that do not usually evoke symptoms. The number of foods the child can eat is then increased by one food per week. In an audit of their results for this mode of treatment, the authors reported a successful outcome in 17 of 22 patients with abdominal migraine, although further study is needed.

Of the nonpharmacological treatment options available, psychotherapy (in particular cognitive behavior therapy) appears to have the most promising outcome. Meta-analysis of well designed studies has shown psychotherapy to be effective in both children and adults with chronic functional abdominal pain. ${ }^{29-31}$ In order to carry out psychotherapy effectively, psychoeducation must first be addressed. This includes an introduction to the concept of pain, epidemiology, and natural history of the disease process and coping strategies including stress reduction. Cognitive behavior therapy can then be used with the idea that pain may not be purely caused by nociception but may also be caused by possible secondary gain, including parental attention and time away from school. ${ }^{8}$

Cognitive behavioral therapy versus standard pediatric care was assessed by Sanders et al. ${ }^{32}$ Forty-four children aged 7-14 years who had recurrent abdominal pain were observed. They were randomly assigned to either the cognitive behavior therapy group or the standard pediatric care group. At 6 months, the cognitive behavior therapy group were pain-free in $55.6 \%$ and $70 \%$ of cases based on diary and parental assessment, respectively. These figures were $58.8 \%$ and $82.4 \%$ at 12 months. The results in this group were significantly better than in the standard pediatric care group, which showed percentages of children pain-free at 12 months of $36.8 \%$ and $42.1 \%$ by diary and parental assessment, respectively. ${ }^{32}$

\section{Pharmacological therapy}

Drug therapy has often been reserved for patients displaying severe, frequent symptoms or those refractory to nonpharmacological therapy. The criteria for selecting children is poorly defined and may be based more on the viewpoint of the families involved than on any objective measure of severity. ${ }^{25}$ The agents available are primarily used due to their proven or presumed activity in migraine headache and for that reason much of the research has been based on serotonin antagonists, beta blockers, or calcium channel inhibitors.

Propranolol was investigated as a treatment for abdominal migraine by Worawattanakul et al. ${ }^{33}$ The authors retrospectively identified 53 patients treated for abdominal migraine over a 6-year period. Twenty-four of these patients were treated with propranolol (10-20 $\mathrm{mg}, 2-3$ times daily). Eighteen $(75 \%)$ of the treated patients had an excellent response with no persistent symptoms. Two patients $(8 \%)$ had a fair response with less frequent or less intense episodes, and four patients (17\%) had no response to the intervention. Of the four children who did not respond to propranolol, two responded to cyproheptadine, a serotonin antagonist. The conclusion stated that, in the majority of patients with abdominal migraine, prophylaxis with propranolol is effective. It is believed that this effect is a direct result of its beta blocking action, but the exact mechanism in unknown. ${ }^{33}$

Serotonin antagonists such as cyproheptadine and pizotifen have also been studied in the treatment of abdominal migraine. In their investigation of propranolol as prophylaxis for abdominal migraine, Worawattanakul et $\mathrm{al}^{33}$ 
studied the effectiveness of cyproheptadine in patients for whom propranolol was contraindicated or ineffective. Of the 12 patients treated with cyproheptadine $(0.25-0.5 \mathrm{mg} /$ $\mathrm{kg}$ day), four patients (33\%) had a complete resolution of symptoms, six patients $(50 \%)$ had a fair response with less frequent or less severe episodes, and two (17\%) patients had no response after 6 months. The authors concluded that most patients with abdominal migraine benefit from prophylactic treatment and that cyproheptadine probably works via its anti-serotonin action. ${ }^{33}$

A double-blind, placebo-controlled trial of pizotifen was carried out by Simon and Russell. ${ }^{34}$ The main antimigraine action of pizotifen is thought to be via serotonin antagonism in addition to its antihistamine properties. The authors of this study recruited 16 patients from a general hospital pediatric clinic with abdominal migraine in whom the duration of pain was at least 2 hours. Additionally, patients were required to have a first-degree or second-degree relative with migraine or recurrent throbbing headaches. The children were given $5 \mathrm{~mL}(0.25 \mathrm{mg})$ of pizotifen twice daily, increased to three times daily if there was no improvement in symptoms. Of the 14 children who completed this trial, 11 patients preferred pizotifen, two preferred placebo, and one was undecided. During treatment with pizotifen, the children had significantly fewer symptoms and their "indices of severity or misery" were reduced. Symon and Russell concluded that pizotifen was successful in treating abdominal migraine in about $70 \%$ of cases and that it would have been unethical to continue the study because pizotifen clearly showed benefit over placebo. They also reported two side effects, in that one child had slight drowsiness and another had increased appetite, although the mean weight gain in treated patients was $1.25 \mathrm{~kg}$ compared with $0.38 \mathrm{~kg}$ in the placebo group. ${ }^{34}$

In addition to the beta-blockers and serotonin antagonists discussed above, the calcium channel antagonist, flunarizine, has also been used successfully in the treatment of childhood and adult migraine. Kothare ${ }^{35}$ performed a retrospective analysis of all patients seen in a neurology clinic in Bombay, India, and isolated 10 children who met the diagnostic criteria for abdominal migraine over a 2-year period. A mean dose of $7.5 \mathrm{mg}$ flunarizine per day was given $(2.5-15 \mathrm{mg})$ and the children were followed up after a mean average of 13 (6-24) months. The data suggested that there was a $61 \%$ reduction in frequency $(0.49 / 0.8)$ and a $51 \%$ reduction in duration $(7.4 / 14)$ of abdominal migraine if prophylaxis with flunarizine was used. The authors concluded that flunarizine is a safe, once-daily option for abdominal migraine prophylaxis. However, they conceded that although its efficacy in this study matches its efficacy in migraine demonstrated in large open US trial, larger studies would need to be conducted to confirm this observation. ${ }^{35}$

Of the pharmacologic treatments available, valproic acid ${ }^{36}$ and sumatriptan ${ }^{37}$ are two of the newer agents to have been studied in relation to abdominal migraine. The authors of studies in which these drugs have been used report successful results; however, this evidence is limited to small case series containing only two patients each. It is thought that these medications may work due to similarities in the underlying pathophysiology of migraine and abdominal migraine, although firm evidence for the etiology of abdominal migraine is lacking. As with all the aforementioned pharmacological therapies, larger and more robust studies are required before a consensus of opinion can be reached.

\section{Conclusion}

The clinical features and prevalence of abdominal migraine in children have been well described in the literature since the early 20 th century. However, recent studies still indicate a trend towards underdiagnosis of this condition despite well defined diagnostic criteria. A recent retrospective study by Carson et $\mathrm{al}^{2}$ of patients referred to a gastroenterology clinic with recurrent abdominal pain found that $4.4 \%$ of patients met the International Classification of Headache Disorders Second Edition criteria for abdominal migraine. A further 11\% had "probable abdominal migraine" based on their failure to meet all the criteria, but being otherwise consistent with the diagnosis. None of the patients reviewed had received a diagnosis of abdominal migraine at that particular practice.

The strong family history of migraine headache often found in children with abdominal migraine should provide physicians with additional supporting evidence in making the diagnosis. Furthermore, the strong association between migraine headache and abdominal migraine should empower physicians to provide reassurance and prognostic information to patients and their parents without the need for invasive costly investigations. The utilization of the modified diagnostic algorithm as suggested may aid physicians in reaching a confident diagnosis of functional abdominal pain without unnecessary tests.

The pathophysiology and treatment of abdominal migraine are perhaps in most need of further investigation and analysis. While the treatment options are varied, they are not based on any proven etiology. If reassurance and cognitive behavior therapy fails, our pharmacological agent of choice is propranolol. We use cyproheptadine as a secondline agent for patients in whom propranolol is ineffective or 
contraindicated. Food exclusion is advised only if there are definitive features to suggest food allergy.

The correct way to treat abdominal migraine most likely involves a combination of different treatments. Physicians will most likely continue to treat abdominal migraine based on their own personal experience until a large randomized control study provides evidence of clear benefit of a particular therapy. Further research is also needed into the etiology of abdominal migraine so that future treatment options may be tailored to the pathophysiology of the condition.

\section{Disclosure}

The authors report no conflicts of interest.

\section{References}

1. Abu-Arafeh I, Russell G. Prevalence and clinical features of abdominal migraine compared with those of migraine headache. Arch Dis Child. 1995;72:413-417.

2. Carson L, Lewis D, Tsou M, et al. Abdominal migraine: an underdiagnosed cause of recurrent abdominal pain in children. Headache. 2011;51:707-712.

3. Mortimer MJ, Kay J, Jaron A. Clinical epidemiology of childhood abdominal migraine in an urban general practice. Dev Med Child Neurol. 1993;35:243-248.

4. Cuvellier JC, Lepine A. Childhood periodic syndromes. Pediatr Neurol. 2010;42:1-11.

5. Cullen KJ, MacDonald WB. The periodic syndrome: its nature and prevalence. Med J Aust. 1963;ii:167-173.

6. Farquhar HG. Abdominal migraine in children. Br Med J. 1956;1: 1082-1085.

7. Olesen J. The International Classification of Headache Disorders, Second Edition. Cephalalgia. 2004;24 Suppl 1:9-160.

8. Bufler P, Gross M, Uhlig HH. Recurrent abdominal pain in childhood. Deutsches Arzteblatt International. 2011;108:295-304.

9. Bremner AR, Sandhu BK. Recurrent abdominal pain in childhood: the functional element. Indian Pediatr. 2009;46:375-379.

10. Bentley D, Kehely A, Al-Bayaty M, Michie CA. Abdominal migraine as a cause of vomiting in children: a clinician's view. J Pediatr Gastroenterol Nutr. 1995;21 Suppl 1:S49-S51.

11. Zeiter DK, Hyams JS. Recurrent abdominal pain in children. J Pediatr Gastroenterol Nutr. 2002;49:53-71.

12. Collins BS, Thomas DW. Chronic abdominal pain. Pediatr Rev. 2007;28:323-331.

13. Brams WA. Abdominal migraine. JAMA. January 7, 1922.

14. Symon DN, Russell G. Abdominal migraine: a childhood syndrome defined. Cephalalgia. 1986;6:223-228.

15. Dignan F, Abu-Arafeh I, Russell G. The prognosis of childhood abdominal migraine. Arch Dis Child. 2001;84:415-418.

16. Rasquin A, Di Lorenzo C, Forbes D, et al. Childhood functional gastrointestinal disorders: child/adolescent. Gastroenterology. 2006;130: $1527-1537$.

17. Helgeland H, Flagstad G, Grotta J, Vandvik PO, Kristensen H, Markestad T. Diagnosing pediatric functional abdominal pain in children according to the Rome III criteria. J Pediatr Gastroenterol Nutr. 2009;49:309-315.
18. Apley J, Naish N. Recurrent abdominal pains: a field survey of 1,000 schoolchildren. Arch Dis Child. 1958;33:165-170.

19. Popovich D, Schentrup DM, McAlhany AL. Recognising and diagnosing abdominal migraines. J Pediatr Health Care. 2010;24:372-377.

20. Devenarayana NM, Rajindrajith S, Janaka De Silva H. Recurrent abdominal pain in children. Indian Pediatr. 2009;46:389-399.

21. Thiessen PN. Recurrent abdominal pain. Pediatr Rev. 2002;23: $39-46$.

22. Catto-Smith AG, Ranuh R. Abdominal migraine and cyclical vomiting. Semin Pediatr Surg. 2003;12:254-258.

23. Lanzi G, Balottin U, Ottolini A, Rosano Burgio F, Fazzi E, Arisi D. Cephalalgia. Cyclic vomiting and recurrent abdominal pains as migraine or epileptic equivalents. $1983 \mathrm{Jun} ; 3(2): 115-118$.

24. Arruda MA, Guidetti V, Galli F, Albuquerque RC, Bigal ME. Childhood periodic syndromes: a population based study. Pediatr Neurol. 2010;43:420-424.

25. Russell G, Abu-Arafeh I, Symon DN. Abdominal migraine: evidence for existence and treatment options. Pediatr Drugs. 2002;4:1-8.

26. Feldman W, McGrath P, Hodgeson C, Ritter H, Shipman RT. The use of dietary fibre in the management of simple, childhood, idiopathic, recurrent abdominal pain. Results in a prospective, double-blind, randomised, controlled trial. Am J Dis Child. 1985;139: 1216-1218.

27. Barr G. Recurrent abdominal pain of childhood due to lactose intolerance - a prospective study. N Engl J Med. 1979;300:1449-1452.

28. Liebman WM. Recurrent abdominal pain in children: lactose and sucrose intolerance, a prospective study. Pediatrics. 1979;64:43-45.

29. Ecclestone C, Palermo TM, Williams AC, Lewandowski A, Morley S. Psychological therapies for the management of chronic and recurrent pain in children and adolescents. Cochrane Database Syst Rev. 2009;2:CD003968.

30. Huertas-Ceballos A, Logan S, Bennett C, MacArthur C. Pharmacological interventions for recurrent abdominal pain and irritable bowel syndrome in childhood. Cochrane Database Syst Rev. 2008;1:CD003017.

31. Palermo TM, Ecclestone C, Lewandowski A, Williams AC, Morley S. Randomised controlled trials of psychological therapies for management of chronic pain in children and adolescents: an updated meta-analytic review. Pain. 2010;148:387-397.

32. Sanders MR, Cleghorn G, Shepherd RW, Patrick M. Predictors of clinical improvement in children with recurrent abdominal pain. Behav Cogn Psychother. 1996;24:26-28.

33. Worawattanakul M, Rhoads MJ, Lichtman SN, Martin H. Abdominal migraine: prophylactic treatment and follow-up. J Pediatr Gastroenterol Nutr. 1999;28:33-40.

34. Symon DN, Russell G. Double blind placebo controlled trial of pizotifen syrup in the treatment of abdominal migraine. Arch Dis Child. 1995; 72:48-50.

35. Kothare SV. Efficacy of flunarizine in the prophylaxis of cyclical vomiting and abdominal migraine. Eur J Paediatr Neurol. 2005;9:23-26.

36. Tan V, Sahami AR, Peebles R, Shaw RJ. Abdominal migraine and treatment with intravenous valproic acid. Psychosomatics. 2006;47: 353-355.

37. Kakisaka Y, Wakusawa K, Haginoya K, et al. Efficacy of sumatriptan in two pediatric cases with abdominal pain-related-functional gastrointestinal disorders: does the mechanism overlap that of migraine? J Child Neurol. 2010;25:234-237. 
Pediatric Health, Medicine and Therapeutics

Dovepress

\section{Publish your work in this journal}

Pediatric Health, Medicine and Therapeutics is an international, peerreviewed, open access journal publishing original research, reports, editorials, reviews and commentaries. All aspects of health maintenance preventative measures and disease treatment interventions are addressed within the journal. Practitioners from all disciplines are invited to submit

Submit your manuscript here: http://www.dovepress.com/pediatric-health-medicine-and-therapeutics-journal their work as well as healthcare researchers and patient support groups. The manuscript management system is completely online and includes a very quick and fair peer-review system. Visit http://www.dovepress. $\mathrm{com} /$ testimonials.php to read real quotes from published authors. 